\title{
Intervenção para stress e ansiedade em pré-vestibulandos: estudo piloto
}

\author{
Carla Cristina Daolio ${ }^{1}$ \\ Carmem Beatriz Neufeld \\ Faculdade de Filosofia, Ciências e Letras de Ribeirão Preto, Ribeirão Preto-SP, Brasil
}

\section{Resumo}

O vestibular se tornou um grande fator de comprometimento da saúde mental dos jovens. O objetivo desse artigo é relatar a experiência de aplicação de um programa breve de intervenção para o manejo de stress e ansiedade em vestibulandos e apresentar seus resultados preliminares. Foram utilizados instrumentos de avaliação psicológica para análise da ansiedade, depressão, desesperança, stress e repertório de habilidades sociais, aplicados aos 12 participantes antes e após a intervenção, composta por seis sessões grupais. Os resultados e relato dos participantes registrado durante as sessões foram promissores, indicando melhora nos sintomas de alguns participantes, embora estes dados devam ser analisados com cautela.

Palavras-chave: stress, ansiedade, vestibular

\section{Abstract: Intervention for stress and anxiety in senior high school students: a pilot study}

The college entrance examination has become a major factor affecting the mental health of young people. The objective of this article is to report the experience of applying a brief intervention program for the management of stress and anxiety in senior high school students and present its preliminary results. Psychological evaluation tools were used to analyze anxiety, depression, hopelessness, stress and repertoire of social skills, applied to the 12 participants before and after the intervention, composed of six group sessions. The results and report of the participants recorded during the sessions were promising, indicating improvement in the symptoms of some participants, although these data should be analyzed with caution.

Keywords: stress, anxiety, entrance examination

\section{Resumen: Intervención para el estrés y la ansiedad en preuniversitarios: estudio piloto}

Las pruebas de ingreso a la educación superior se han convertido en un gran factor de riesgo para la salud mental de los jóvenes. El objetivo de este artículo es relatar la experiencia de aplicación de un breve programa de intervención para el manejo del estrés y la ansiedad en estudiantes preuniversitarios y presentar sus resultados preliminares. Se utilizaron instrumentos de evaluación psicológica para el análisis de la ansiedad, depresión, desesperanza, estrés y repertorio de habilidades sociales, aplicados a los 12 participantes, antes y después de la intervención, que estuvo compuesta por seis sesiones grupales. Los resultados y manifestaciones de los participantes registrados durante las sesiones fueron promisores, indicando mejoría en los síntomas de algunos participantes, aunque estos datos deben ser analizados con cautela.

Palabras clave: estrés, ansiedad, pruebas de ingreso

\footnotetext{
${ }^{1}$ Endereço para correspondência: Rua Rafael Sampaio, 500, 13023-240, Vila Rossi, Campinas, SP. E-mail: carlinhaccd@gmail.com
} 
Este artigo é resultado de uma experiência inicial envolvendo a intervenção para manejo de stress e ansiedade com vestibulandos. A partir da demanda encontrada nesta população, foi proposta a construção de um programa de intervenção, baseado na Abordagem CognitivoComportamental, cuja aplicação piloto e resultados preliminares serão apresentados e discutidos.

Contextualizando o problema, o vestibular é uma prova concorrida, classificatória, difícil e exigente, na qual o aluno deve ser capaz de aplicar todo o seu conhecimento adquirido na trajetória escolar e, ao mesmo tempo, manter o controle emocional (D'Avila \& Soares, 2003). Sua complexidade, assim como a dificuldade de acesso, são características da educação superior no Brasil, principalmente nas universidades públicas, visto o reduzido número de vagas em relação ao volume de pleiteantes às mesmas (D'Avila, Krawulski, Veriguine, \& Soares, 2011).

O vestibular passa a ser visto como ritual de passagem, a partir do qual o adolescente entrará no mundo adulto e do trabalho (Dias, Nazareno, Zanini, \& Mendonça, 2008; Fagundes, Aquino, \& Paula, 2010; Paggiaro \& Calais, 2009; Rodrigues \& Pelisoli, 2008), sendo que a época que o antecede caracteriza-se como uma fase de turbulência e insegurança. Muitas vezes a prova contribui para o surgimento de stress e psicopatologias como ansiedade e depressão (Dias et al., 2008), visto que a grande pressão que exerce no estudante é acompanhada pelo medo do fracasso e das escolhas malsucedidas, pela disputa de vagas, pelo pouco tempo de preparo, pela sensação de que se falharem terão perdido todo o ano de estudos (Paggiaro \& Calais, 2009), pela pressão e expectativas da família, de amigos e da sociedade (Dias et al., 2008; Rodrigues \& Pelisoli, 2008).

A prova torna-se mais uma fonte de comprometimento, já que ela é a maneira de alcançar seu futuro profissional, gerando ansiedade e um estado de tensão e stress emocional (Guhur, Alberto, \& Carniatto, 2010). As referidas autoras relembram a partir da literatura que eventos estressores podem ser dependentes ou independentes. Nesse sentido, ainda de acordo com Guhur, Alberto e Carniatto (2010), o vestibular seria um evento independente, já que o adolescente não tem controle sobre ele. Já os eventos dependentes estão relacionados à maneira com que o indivíduo irá agir diante de uma situação potencialmente estressora, como o vestibular por exemplo. No mesmo sentido, Silva e Zanini (2011) denotam que não apenas a vivência dos eventos estressores afeta os vestibulandos, mas também a forma como os enfrentam, interferindo no aumento ou diminuição das suas consequências. $\mathrm{O}$ estado emocional e psicológico no momento da prova, como o sentimento de obrigação em prestá-la e o fato de ela ser considerada decisiva, afetam muito a ansiedade do vestibulando, que por muitas vezes, em decorrências desses fatores, acaba não sendo aprovado (Guhur et al., 2010).

A questão da escolha profissional também parece interferir no bem estar dos jovens nessa etapa da vida. $\mathrm{O}$ vestibular inevitavelmente remete à mesma, que é multifatorial, envolvendo aspectos políticos, econômicos, sociais, educacionais, familiares e psicológicos (Fagundes et al., 2010; Paggiaro \& Calais, 2009). A escolha pode motivar o adolescente a estudar e seguir um planejamento, ou pode se tornar um fator ansiogênico devido à necessidade de conhecimento da área de atuação, mercado de trabalho, rotina, salário e todos os acompanhantes da vida profissional (Rodrigues \& Pelisoli, 2008). Dessa forma, o auxílio ao jovem nas decisões de carreira promove melhora no desempenho acadêmico, na motivação e na sensação de bem estar, contribuindo para uma melhor qualidade de vida (Paggiaro \& Calais, 2009).

Analisando um cursinho popular vinculado a uma universidade federal, D'Avila et al. (2011) buscaram identificar as repercussões desse cursinho no projeto profissional, ligado à questão da identidade humana dos estudantes, segundo suas perspectivas e adotando metodologia qualitativa. Quanto às expectativas em relação ao futuro, os resultados indicaram que os estudantes têm dificuldade de antecipar situações profissionais e de relacionar as emoções do contexto no momento atual com o momento futuro. Percebeu-se um desejo de "ser alguém" atrelado e dependente da aprovação no vestibular; mas, ao mesmo tempo, singular a cada participante visto as diferentes histórias pregressas que também influenciam nessas expectativas. Em relação ao projeto profissional em si, para todos os participantes ele estava atrelado aos estudos, ou seja, a primeiro passar no vestibular, para que depois pudessem concluir o ensino superior e então poder ter uma profissão que lhes permitissem ser alguém no mundo.

A fim de investigar os efeitos que o vestibular gera na vida dos adolescentes, Guhur et al. (2010) realizaram uma pesquisa qualitativa, através da análise das respostas a um questionário por 37 participantes de ambos os sexos e de escola pública e particular. Os resultados mostraram que a preferência foi por graduação em universidades públicas, visto considerarem serem de melhor qualidade e de maior acesso ao mercado de trabalho, ou por questões financeiras. As escolhas pelos cursos foram moldadas por afinidade (vinte e cinco alunos), pelo curso "dos sonhos" (quinze alunos), pela influência da família (três alunos) e pela possibilidade de retorno financeiro da profissão (seis alunos). A maioria dos pais apoiava a escolha do curso dos filhos. Entretanto, $65 \%$ dos participantes (principalmente as meninas e os alunos de escola 
particular) relataram sentirem-se pressionados pelos pais, indiretamente, através de comparações com irmãos que já haviam sido aprovados, por exemplo. Dentre os participantes, $76 \%$ relataram sentirem-se nervosos, angustiados e ansiosos por não saberem como é o vestibular, pela primeira vez, ou por acharem que o tempo para preparação é muito curto. Outras preocupações relacionaram-se ao medo de esquecerem-se da matéria no dia. Quase todos afirmaram temer o resultado da prova, por questões familiares, pela prova ser decisiva para o futuro, por não aguentarem estudar mais e por demorarem a achar um emprego. Sobre o que fariam caso não passassem no vestibular, todos os participantes das escolas particulares disseram que fariam cursinho; enquanto alguns da escola pública fariam cursinho, outros começariam a trabalhar ou prestariam concurso.

Em contraponto a este resultado, um estudo conduzido na Finlândia encontrou que estudantes que não obtiveram aprovação no exame de seleção para entrada na universidade tiveram níveis de satisfação com a vida mais altos em associação com o uso de estratégias de controle compensatório secundário, sugerindo que abandonar o objetivo de passar no exame poderia ser mais adaptativo (Tomasik \& Salmela-Aro, 2012). Na realidade brasileira, como se percebe pelo estudo de Guhur et al. (2010), esta não parece ser a opção mais desejada pelos vestibulandos, que em sua maioria continuam tentando o ingresso na universidade.

Com o intuito de avaliar as percepções que os vestibulandos têm do processo seletivo, Fagundes et al. (2010) realizaram uma pesquisa qualitativa com 20 adolescentes que prestariam o vestibular. Várias informações foram extraídas das entrevistas semiestruturadas. O acúmulo de atividades provocava desânimo nos participantes, sentindo-se pressionados e sobrecarregados, com menor entusiasmo para realizar outras tarefas. Esse cansaço gera dificuldade em assimilar corretamente o conhecimento, gerando aumento da competitividade e ansiedade. Os momentos que antecedem as provas escolares são de muita tensão, mas é no período de realização do vestibular em si que as cobranças e tensões são extremas. Os participantes experimentaram insônia, tensão, irritabilidade, desânimo, dificuldade de aprendizagem e impaciência. Quanto às estratégias de enfrentamento, alguns preferiam ficarem quietos ou sozinhos, outros tentavam se distrair, outros tentavam relaxar e alguns tentavam esquecer da situação em que estavam. Os autores ressaltaram o quanto esses jovens poderiam estar necessitando de uma intervenção, para que passassem a utilizar estratégias que tragam benefícios mais específicos em relação ao stress.
A partir de todas as informações que foram apresentadas até aqui é possível identificar que algumas variáveis psicológicas perpassam a questão do vestibular. $\mathrm{O}$ processo pode gerar ansiedade, depressão e stress como foi apontado pela literatura. Essas variáveis, por sua vez, interferem novamente no processo, dificultando a preparação para a prova ou o desempenho durante a própria prova. Além dessas variáveis, outros fatores parecem afetar no momento da prova, como o viés cognitivo de cada estudante e o repertório de habilidades sociais para resolver problemas e utilizar estratégias de coping.

\section{Saúde mental do vestibulando: Stress e variáveis associadas}

Pellegrini, Calais e Salgado (2012) ressaltam a importância de estudos sobre variáveis que controlam o stress para que se conheça os estímulos estressores e as maneiras de enfrentá-los eficazmente. Em Teresina (Araújo et al., 2012) e em Campinas (Souza, Bonganha, Chacon-Mikahil, \& Madruga, 2013), programas de exercício físico têm sido desenvolvidos para auxiliar na redução do stress em vestibulandos e alunas do primeiro ano de faculdades, respectivamente. Os dois programas contaram com uma atividade planejada de exercícios físicos específicos e com a medição dos níveis de stress dos participantes. Em ambas as experiências os níveis de stress que estavam altos a princípio, foram reduzidos.

Em outro estudo, Paggiaro e Calais (2009) discutiram as manifestações do stress em alunos de cursinho pré-vestibular e sua relação com a escolha profissional. O Inventário de Stress de Lipp (ISSL) e a Ficha de Informações sobre Escolha mostraram que $67,7 \%$ dos participantes apresentavam stress, $42,1 \%$ possuíam pouca informação sobre a profissão escolhida, $51,6 \%$ possuíam uma opção de escolha e 64,6\% estavam inseguros quanto à escolha. Na amostra houve predominância dos sintomas psicológicos do stress onde o sintoma mais citado foi o pensamento recorrente em um só assunto. Mais da metade da amostra relatou estar preocupada com o vestibular e o stress pareceu ser maior em estudantes inseguros quanto à escolha profissional.

Em extensa tese, Marques (2014) investigou o estado de stress e os hábitos de vida de estudantes pré-vestibulandos. Participaram 68 estudantes frequentando o terceiro ano do ensino médio e um cursinho pré-vestibular. O ISSL indicou que $76,47 \%$ dos participantes apresentaram stress, sendo a sua maioria na fase de resistência e com predominância de sintomas psicológicos. Dentre os resultados estatisticamente significativos, o autor encontrou predominância de stress no sexo feminino e nos participantes 
insuficientemente ativos de acordo com o Questionário Internacional de Atividade Física (IPAQ-versão curta). Os resultados confirmaram a necessidade de atenção aos estudantes que estão na fase de prestar o vestibular e a importância da manutenção de atividade física como maneira de manejar os sintomas de stress.

A capacidade de responder ao estressor irá depender da percepção que o indivíduo tem de seu meio e de sua habilidade para lidar com ele; em outras palavras, da interpretação cognitiva ou do significado que o indivíduo atribuirá ao estressor (Fagundes et al., 2010). Se não tiver estratégias para lidar com o stress, ele terá dificuldade para exibir o seu conhecimento acadêmico no vestibular (Paggiaro \& Calais, 2009).

A ansiedade é outra variável que afeta a qualidade de vida dos vestibulandos. Kuntze (2013), por exemplo, constatou em alunos de cursinho de Florianópolis que a ansiedade esteve associada à presença de dor de cabeça $\mathrm{e}$ dor ou cansaço facial, sendo que a maioria apresentou ansiedade moderada. Archer, Heumann e Luz Filho (2011) avaliaram relatos relacionados à ansiedade quanto à escolha profissional e quanto ao vestibular, em participantes de um serviço de Orientação Profissional. As entrevistas mostraram que o vestibular se torna um grande gerador de ansiedade, visto o significado de fracasso pessoal atribuído a não aprovação, e ao fato de forçar uma escolha definitiva quando os alunos têm pouco conhecimento ainda sobre as profissões.

Em Campina Grande, Santos (2011) verificou que os níveis de ansiedade aumentaram conforme se aproximava a data do exame, gerando aumento no consumo de açúcares, no peso, no IMC e na circunferência abdominal dos estudantes, havendo prejuízo em sua saúde. Lins e Viana (2016) também verificaram aumento da ansiedade em alunos que se aproximavam da data da realização do vestibular, bem como má qualidade do sono com consequente uso negligente de psicotrópicos. Confirmando esses dados, Rodrigues e Pelisoli (2008) constataram que dentre 1046 jovens que prestariam o vestibular, mais de $90 \%$ declararam que seus hábitos de vida foram alterados com a preparação para a prova. Relacionamentos e atividades como sono e alimentação passaram a funcionar de maneira diferente. $\mathrm{O}$ processo seletivo maximizou a ansiedade inerente às questões da adolescência e esteve presente em todos os participantes, independente de outras questões como carreira, pressão da família, entre outros. Fatores que contribuíram para a formação e a manutenção da ansiedade foram: a espera e a preparação até o dia da prova, o fato de o processo ser competitivo, a ideia de que somente os melhores são aprovados, e o fato de o sucesso depender do desempenho dos outros candidatos e não somente do próprio esforço.
Apesar de a literatura mencionar que o processo vestibular pode contribuir para o aparecimento de depressão, não foram encontradas muitas pesquisas que tenham estudado especificamente e de modo aprofundado essa relação. O mesmo acontece com as habilidades sociais, embora elas tenham aparecido de forma indireta nas pesquisas referentes a estratégias de enfrentamento (Kristensen et al., 2010; Silva \& Zanini, 2011), ou a habilidades sociais e sua relação com o stress (Pellegrini et al., 2012). Considerando esse fato como uma lacuna na literatura, incentiva-se o estudo dessas variáveis (depressão e habilidades sociais) na população de vestibulandos. Isto porque a aquisição de habilidades sociais amplia a percepção do indivíduo sobre sua própria competência, promovendo autoconfiança para responder adequadamente aos estressores sociais, se tornando variáveis importantes no controle do stress (Pellegrini et al., 2012).

Todas as informações e discussões apresentadas até aqui demonstram que a população do estudo, os vestibulandos, podem necessitar de intervenção psicológica. A literatura apontou para a necessidade de várias intervenções importantes que devem ser realizadas com essa população, visto que muitos estudantes têm o conhecimento acadêmico necessário, mas não conseguem expressá-lo devido à inabilidade de manejar o stress e a ansiedade (Guhur et al., 2010). Maria, Guimarães e Matias (2009), por exemplo, comentam sobre exercícios de relaxamento, análise de experiências de sucesso e seus resultados positivos para o manejo de ansiedade, além da prática de atividade física para combater o stress.

Embora Rodrigues e Pelisoli (2008) relatem que têm sido oferecidos grupos terapêuticos que buscam aliviar a ansiedade e preparar emocionalmente os alunos de curso pré-vestibular em Porto Alegre, denota-se uma escassez de publicações científicas de programas estruturados com essa finalidade. Mais específicos para alunos que estão prestando vestibular, encontra-se livros destinados aos vestibulandos que tratam de questões relacionadas, como a escolha da profissão e o preparo emocional e acadêmico para a prova (Villamarín, 2000). O presente estudo busca suprir esta lacuna, descrevendo os achados preliminares de uma experiência piloto, cujo mote foi a construção de um programa de intervenção para manejo de stress e ansiedade causados pelo vestibular, baseado na Abordagem Cognitivo-Comportamental.

Considerando que a percepção do vestibulando afeta a maneira como ele lidará com os estressores do processo seletivo e que pensamentos ou crenças disfuncionais que os vestibulandos têm em relação ao vestibular afetam o seu desempenho negativamente, a Terapia Cognitiva Comportamental (TCC) pode auxiliar o jovem neste 
período. Isto em razão de a TCC ter como princípio fundamental que a maneira como as pessoas percebem e processam a realidade influenciará diretamente a maneira como elas se sentem e se comportam (Knapp \& Beck, 2008). Dessa maneira, a TCC se propõe a promover a reestruturação cognitiva desses pensamentos e, colaborativamente, desenvolve soluções pragmáticas para mudar e melhorar transtornos emocionais (Knapp \& Beck, 2008).

Sob a luz da TCC, Caciari e Damazo (2017) refletem sobre o uso de estratégias de enfrentamento de um pequeno grupo de jovens em situação de vulnerabilidade social. Chegaram à conclusão que apesar de as estratégias utilizadas terem sido dependentes das características de personalidade de cada jovem, o contexto social e o processo de desenvolvimento devem ser distintamente considerados para entender as escolhas das estratégias de enfrentamento. Dessa forma, em consonância com os princípios da TCC, as autoras consideraram que independentemente da situação na qual o adolescente se encontre, o que afetará sua maneira de enfrentamento será o modo como ele a interpreta.

As contribuições da abordagem também ficam evidentes no estudo de Oliveira, Dias e Piccoloto (2013) sobre as dificuldades de adaptações acadêmicas. Com a participação de 504 estudantes de diferentes universidades do Sul do Brasil, os autores perceberam que a TCC tem escopo teórico e metodológico tanto para compreender como para intervir nas dificuldades relatadas, se constituindo numa boa ferramenta para auxiliar estudantes na transição para o ensino superior.

Considerando casos de uma clínica-escola do interior de Minas Gerais, Moreno e Carvalho (2014) avaliaram o efeito de intervenções breves em TCC em sintomas de depressão e ansiedade. Através da comparação dos resultados em instrumentos validados antes e após as intervenções, os autores observaram melhoras significativas nos sintomas dos pacientes. Em relação ao tratamento para stress, Lipp (2017) descreve o treino psicológico de controle de stress (TCS) como um modelo de intervenção grupal desenvolvido no Laboratório de Estudos Psicofisiológicos do Stress da PUC-Campinas. Baseado em pesquisas da área o protocolo teve sua eficácia comprovada em várias pesquisas, dissertações de mestrado, teses de doutorado e trabalhos de conclusão de pós-doutorado.

Mesmo dentro da abordagem, a literatura para intervenção com a população específica de pré-vestibulandos é escassa, apesar de vários estudos evidenciarem sua eficácia com diferentes idades e sintomas. Os estudos citados demonstram que os princípios norteadores e práticas da TCC são compatíveis para o desenvolvimento de uma intervenção de qualidade sobre o stress e a ansiedade desses alunos.

\section{Método}

A oportunidade para a aplicação de um programa piloto surgiu da demanda de alunos de um cursinho pré- vestibular particular de uma cidade do interior de São Paulo. A instituição de ensino, bem como os alunos, demonstrou interesse em participar da experiência após saberem do projeto através do contato entre um profissional da instituição e a pesquisadora. Com a aprovação do Comitê de Ética em Pesquisa da Faculdade de Filosofia, Ciências e Letras de Ribeirão Preto (FFCLRP-USP) ( ${ }^{\circ}$ de aprovação: 957.682), conforme estipulado pela Resolução 466/2012 do Conselho Nacional de Saúde, os alunos foram convidados a participar do programa em sala de aula. Nas semanas seguintes, com os estudantes que desejaram participar, foi dado início ao projeto que se constituiu na aplicação de um pré-teste, seis sessões de intervenção (uma por semana), um pós-teste e devolutivas individuais.

\section{Participantes}

Interessaram-se pela participação em uma primeira experiência com o programa 12 adolescentes de ambos os sexos e idades entre 17 e 21 anos (média de 18,33 e desvio padrão de 1,45). Todos eram membros de uma mesma turma de cursinho pré-vestibular privado. O curso pretendido pela maioria foi Medicina (sete alunos), seguido por Odontologia (dois alunos) e empatados com um aluno cada um, Relações Internacionais e Direito, havendo uma abstenção. Dois alunos estariam prestando a prova pela primeira vez, cinco pela segunda vez, quatro pela terceira vez e um pela quarta vez.

\section{Instrumentos}

As Escalas Beck na versão traduzida e validada para a população brasileira (Cunha, 2001) compõem-se por um conjunto de quatro escalas desenvolvidas por Beck, que avaliam ansiedade, depressão, desesperança e ideação suicida. Neste estudo foram utilizadas as escalas de ansiedade, depressão e desesperança por serem essas as variáveis referidas pela literatura a interferir no processo seletivo do vestibular. Podem ser aplicadas a indivíduos de 17 a 80 anos. O Inventário de Ansiedade de Beck (BAI), o Inventário de Depressão de Beck (BDI) e a Escala de Desesperança de Beck (BHS) avaliam a gravidade dos sintomas das respectivas variáveis que medem, apontando para níveis mínimos, leves, moderados ou severos para ansiedade e depressão, e para níveis mínimos, leves, moderados ou graves de desesperança. 
Para a avaliação do stress, foi utilizado o Inventário de Sintomas de Stress de Lipp (ISSL) (Lipp, 2000). O instrumento é composto por três quadros que avaliam as quatro fases do stress (alerta, resistência, quase-exaustão e exaustão) e os sintomas do stress (físicos e psicológicos). Pode ser aplicado a respondentes que tenham no mínimo 15 anos.

Já a avaliação das habilidades sociais baseou-se no Inventário de Habilidades Sociais (IHS) (Del Prette \& Del Prette, 2001). Conforme indicações do manual, esse instrumento tem sido utilizado para a mensuração das habilidades de adultos com formação de segundo e terceiro graus, adolescentes e pré-vestibulandos. A escala avalia, a partir de pontuações de acordo com as respostas e seus respectivos percentis, o repertório geral de habilidades sociais e os repertórios em cinco fatores específicos: enfrentamento e autoafirmação com risco, autoafirmação na expressão de sentimento positivo, conversação e desenvoltura social, autoexposição a desconhecidos e situações novas, autocontrole da agressividade.

\section{Programa}

Apoiando-se nas informações reunidas na revisão de literatura, o programa foi elaborado a partir de intervenções propostas pela TCC e do Treino de Controle de Stress (Lipp, 2017). Constituiu-se de seis sessões com cerca de 50 minutos de duração cada, que versaram sobre os temas: apresentação e psicoeducação sobre stress, ansiedade e sua relação com o vestibular; psicoeducação sobre hábitos saudáveis (alimentação e exercícios físicos); técnica de relaxamento físico e mental e de respiração; psicoeducação do modelo cognitivo; treino de identificação de pensamentos relacionados ao stress e à ansiedade; psicoeducação e identificação de distorções cognitivas; intervenção cognitivo-comportamental para pensamentos geradores de ansiedade e stress (questionamento socrático; descatastrofização, resolução de problemas, exposição, entre outros); fechamento e avaliação qualitativa. Durante as sessões de intervenção, os relatos emitidos pelos participantes sobre os temas das mesmas e suas opiniões foram utilizados para complementação das análises dos resultados e dos efeitos do programa.

O espaço de realização das sessões, bem como das testagens, foi cedido pela escola dos estudantes. A aplicação dos instrumentos foi realizada coletivamente em sala de aula e as sessões foram conduzidas em duas outras salas, com espaço e estrutura mais adequados às atividades em grupo, de acordo com a disponibilidade das mesmas, a depender das atividades realizadas na instituição. A duração dos encontros ficou em torno de 50 minutos e foi definida pela disponibilidade dos alunos e negociações com a instituição, visto sua ampla grade horária. Os grupos aconteciam no horário de almoço dos estudantes, após acordos para que ele pudesse ser estendido, permitindo a participação no grupo sem prejuízo às aulas.

\section{Resultados e Discussão}

\section{Avaliação inicial}

A avaliação inicial dos participantes, através da aplicação dos instrumentos, cumpriu objetivos diagnósticos para efeito de nortear a intervenção e possibilitar, ao fim da mesma, a análise de seus efeitos nos parâmetros avaliados. Os resultados no pré-teste mostraram que a maioria dos alunos de fato estava apresentando sinais de desgaste psicológico, principalmente stress e ansiedade, apesar de possuírem bom repertório de habilidades sociais.

Para melhor entendimento sobre os resultados da análise de stress, Paggiaro e Calais (2009) apontam que tradicionalmente ele foi dividido em três fases (Alerta, Resistência, Exaustão), havendo a inclusão de uma fase intermediária (Quase-Exaustão). Essas fases vão crescendo em gravidade à medida que o organismo vai deixando de conseguir lidar com os eventos estressores e se desequilibra, de forma que nas duas últimas fases já há alterações no funcionamento do sistema imunológico e o aparecimento de doenças. O ISSL apontou que apenas dois participantes não apresentavam sinais de stress anteriormente à intervenção, três encontravam-se na fase de quase-exaustão e o restante dos alunos encontrava-se na fase de resistência.

Quanto aos níveis de ansiedade, a BAI apontou que quatro participantes apresentavam níveis mínimos de ansiedade, dois apresentaram ansiedade leve, cinco apresentavam níveis moderados de ansiedade e um participante apresentava ansiedade severa antes do início da intervenção. A BDI mostrou que quatro participantes apresentavam níveis mínimos de sintomas depressivos e cinco apresentavam níveis leves. Entretanto, dois alunos evidenciaram níveis moderados e um apresentou níveis severos de sintomas depressivos. Nenhum participante apresentou níveis moderados ou graves de desesperança segundo a BHS, tendo sido em sua maioria (oito alunos) níveis mínimos.

Finalmente, o IHS apontou que a maioria dos estudantes possuía bom repertório ou repertório elaborado de habilidades sociais. Apenas dois foram identificados como podendo se beneficiar de intervenção com treino em habilidades sociais.

No que tange ao stress e à ansiedade, percebe-se que os resultados do pré-teste parecem condizer com o que a 
literatura tem apontado. Durante a aplicação dos dois programas de exercício físico para redução de stress citados (Araújo, et al., 2012; Souza, et al., 2013) foram constatados altos níveis de stress anteriormente à intervenção. Também no estudo de Paggiaro e Calais (2009), a qual relacionou o stress com o momento de vida da escolha de carreira e o vestibular, grande parte da amostra apresentava stress e esse parecia ser maior em alunos indecisos quanto à carreira. O estudo de Marques (2014) sobre o estado de stress e hábitos de vida em pré-vestibulandos também apresentou alta incidência de stress na amostra. Da mesma forma, a ansiedade foi associada à mudança nos hábitos alimentares conforme a aproximação da data do exame no estudo de Santos (2011). Relatos de aumento de ansiedade relacionados diretamente ao vestibular ou a escolha de carreira foram constatados por Rodrigues e Pelisoli (2008) e por Archer et al. (2011). Aumento da ansiedade frente ao exame e dificuldade de manter qualidade do sono, levando ao uso negligente de psicotrópicos, foi verificado em estudo de Lins e Viana (2016).

Considerando que se tem constatado o aumento de depressão nos adolescentes nos últimos anos (Resende, Santos, Santos, \& Ferrão, 2013), assim como a sua significativa relação com a ideação suicida (Moreira \& Bastos, 2015), a investigação dessa variável em adolescentes não deve ser subestimada. O fato de três alunos $(25 \%$ dos participantes) terem apresentado níveis moderados a severos de depressão chama a atenção, incentivando-se o aumento de estudos que busquem entender melhor os fatores associados ao aumento desses índices, e se o stress e a ansiedade causados pelo vestibular podem de alguma forma estar contribuindo para o aumento de sintomas depressivos. Quanto às habilidades sociais, apesar de estudos mostrarem que elas podem ser afetadas no momento do vestibular (Carrara \& Betetto, 2009), e que a sua presença auxilia no controle do stress (Pellegrini et al, 2012), nesta amostra específica os repertórios de habilidades sociais mostraram-se altos. Tal dado parece indicar que, para esses estudantes em questão, o bom repertório de habilidades sociais não é suficiente para a diminuição dos sintomas de stress e ansiedade, o que mais uma vez justifica a necessidade de intervenção específica para esses sintomas em alunos que estão prestando vestibular.

\section{A intervenção piloto: detalhamento e observações}

A primeira sessão do programa foi destinada à psicoeducação sobre o stress e a ansiedade e a sua relação com o vestibular. A terapeuta explicou, com auxílio de recursos audiovisuais, as funções evolutivas da ansiedade e do stress, salientando que a partir do momento em que se tornam muitos, intensos ou frequentes é que passam a ser prejudiciais à saúde mental e ao desempenho. Também foi explorada a relação que o vestibular tem com esses sintomas e apresentado o planejamento do programa completo. A sessão contou com 10 participantes, os quais tiveram a chance de tirar dúvidas e compartilhar questões pessoais que se relacionavam ao tema. Os jovens foram bastante participativos, relatando que não haviam refletido sobre os conceitos de stress e ansiedade, da forma como foram apresentados, e que reconheciam os sofrimentos que o processo do vestibular os estava causando. Também ressaltaram que não tinham mais dúvidas quanto à escolha de carreira, e que esse não seria um tema que precisaria ser trabalhado em outras sessões.

A sessão seguinte contou com oito participantes e seu objetivo foi a psicoeducação sobre a importância da manutenção de hábitos saudáveis para o combate e controle do stress. Foi discutida com os jovens a sua grade horária, que deveria incluir, além das atividades acadêmicas, tempo para atividades físicas e de lazer, bem como cuidados com a alimentação. Os participantes compartilharam algumas de suas experiências pessoais sobre o assunto, ressaltando algumas dificuldades como a de diminuir o consumo de cafeína e a de manter um sono de qualidade. Foram discutidas em grupo possíveis atitudes que eles mesmos poderiam tomar em sua rotina para lidar com essas dificuldades.

A terceira sessão incluiu exercícios de relaxamento físico e mental e exercícios de respiração. Contando com nove participantes presentes, foram realizados os relaxamentos sugeridos no livro Relaxamento para todos (Lipp e cols, 2012), direcionados ao relaxamento geral, que podem ser utilizados em qualquer situação de stress e direcionados à situação específica do vestibular. O espaço contribuiu bastante para essa sessão, já que os alunos puderam se deitar ou sentar confortavelmente enquanto realizavam os exercícios. O feedback foi bastante positivo no relato dos jovens ao final dessa sessão.

Algumas dificuldades ocorreram na quarta sessão, como o atraso para seu início devido a condições operacionais (problemas no equipamento multimídia) e queda no número de participantes (seis participantes). Apesar do tempo reduzido, foi realizada psicoeducação sobre o Modelo Cognitivo, como parte da intervenção segundo a TCC. Foi explicado como a maneira de interpretar as situações de vida podem interferir nos pensamentos, sentimentos e atitudes, e como essa maneira vem de crenças profundas acerca de si mesmos, dos outros, do mundo e do futuro. Relacionou-se, brevemente, a maneira de encarar as pressões do vestibular com estas crenças. Os participantes presentes foram convidados a prestar 
atenção aos seus pensamentos e sentimentos acerca do vestibular no decorrer das semanas seguintes.

Entre a quarta e quinta sessão houve mudança do dia em que estavam ocorrendo os grupos e tal fato acabou gerando desencontro de informações entre a coordenação e os estudantes, que acabaram se esquecendo da alteração da referida data. Por conta do ocorrido, a quinta sessão contou com apenas três participantes. A sessão versou sobre as distorções cognitivas que levam a interpretações enviesadas dos fatos, que muitas vezes geram comportamentos desadaptativos para lidar com um problema. Foi feito um exercício de identificação de pensamentos, sentimentos e comportamentos em situações comuns que vestibulandos vivenciam, bem como a procura por possíveis distorções de pensamento nessas situações. Para cada uma delas foram discutidas maneiras de lidar com as angústias e preocupações geradas. Os alunos presentes foram instruídos a entregar o material que foi distribuído nesta sessão para os colegas que se ausentaram.

A última sessão teve tempo estendido para que os alunos faltantes tivessem a oportunidade de realizar o mesmo exercício da quinta sessão. Com sete presentes, a terapeuta retomou os conceitos até ali discutidos e abriu para que os próprios jovens incluíssem outros temas que gostariam de trabalhar. Foram levantadas algumas questões sobre como se preparar para o momento da prova em si e em como lidar com possíveis dificuldades (muita preocupação, brancos, atrasos). Para cada situação levantada foi feita em conjunto a técnica de resolução de problemas e foram relembrados os exercícios de relaxamento propícios ao momento da prova. Ao fim da sessão foi pedido aos jovens que expressassem suas opiniões sinceras sobre o programa, que temas mais lhe ajudaram e o que consideravam que estava faltando. Comentaram que estavam tão carregados com a pressão do vestibular que a iniciativa com certeza foi benéfica, destacando-se a sessão com os exercícios de relaxamento. Relataram que a oportunidade de ter um tempo para conversar exclusivamente sobre suas angústias ajudou a aliviar um pouco da pressão que estavam sentindo. Dificuldades foram também mencionadas por alguns participantes: estarem presentes no horário do grupo e a consequente dificuldade de concentração por aquele ser, anteriormente, um horário de descanso. Outro apontamento relevante foi o de que houve pouco tempo para assimilar conteúdos novos, e por vezes angustiantes, ressaltando o incentivo à maior quantidade de sessões.

\section{Avaliação posterior à intervenção: possíveis efeitos}

Terminada a intervenção, os alunos mais uma vez responderam aos instrumentos de avaliação utilizados na pesquisa. Vale ressaltar que logo antes do pós-teste a primeira fase de alguns vestibulares já haviam ocorrido. Realizaram o pós-teste apenas 11 dos 12 participantes.

Os resultados do ISSL indicaram que dois participantes aumentaram a fase de stress à época do pós-teste (um passando da fase de resistência para a de quase-exaustão e um que não apresentava stress para a fase de resistência), dois se mantiveram no mesmo nível (um na fase de resistência e outro continuou não apresentando stress) e os demais apresentaram redução de sintomas (7 participantes). Dentre os que apresentaram queda no nível de sintomas, três reduziram inclusive a fase do stress, passando da quase-exaustão para a de resistência em dois casos, e um para ausência de stress. O Quadro 1 facilita a visualização das mudanças de fase de stress de cada participante, de acordo com o ISSL.

Quadro 1

Fases do Stress no Pré e Pós Teste

\begin{tabular}{ccc}
\hline \multirow{2}{*}{ Participantes } & Fases do Stress \\
\cline { 2 - 3 } & & \\
\hline 1 & Pré-teste & Pós-Teste \\
2 & Resistência & Quase-exaustão \\
3 & Quase-exaustão & Resistência \\
4 & Resistência & Resistência \\
5 & Quase-exaustão & Resistência \\
6 & Quase-exaustão & Não apresenta stress \\
7 & Resistência & Resistência \\
8 & Resistência & Resistência \\
9 & Resistência & Resistência \\
10 & Não apresenta stress & Não apresenta stress \\
11 & Resistência & Resistência \\
\hline
\end{tabular}


Quanto à ansiedade, a BAI indicou que apenas um participante que apresentava ansiedade mínima passou apresentar ansiedade leve, aumento possivelmente relacionado à primeira fase de vestibulares que antecedeu o pós-teste. Dentre os demais, cinco mantiveram níveis leves ou mínimos e cinco reduziram seus níveis de ansiedade, sendo que três passaram de ansiedade moderada para leve, um passou de ansiedade moderada para mínima e um passou de ansiedade severa para ansiedade moderada. Já a BDI mostrou que os três participantes que apresentavam níveis altos de depressão tiveram redução nos sintomas, sendo que o participante com nível severo passou a apresentar nível moderado e os dois que apresentavam nível moderado passaram a apresentar níveis leves. Os demais participantes mantiveram seus níveis do pré-teste. A medição de desesperança concedida pela BHS permitiu observar que se manteve os índices baixos, sendo que dois participantes, que no pré-teste apresentavam níveis leves, passaram a apresentar níveis mínimos. A fim de facilitar a visualização das mudanças após a intervenção, o Quadro 2 mostra o nível de severidade dos sintomas de ansiedade, depressão e desesperança dos participantes, de acordo com as Escalas Beck, antes e após as sessões.

Quadro 2

Nivel de Severidade dos Sintomas de Ansiedade, Depressão e Desesperança no Pré e Pós Teste

\begin{tabular}{|c|c|c|c|c|c|c|}
\hline \multirow{3}{*}{ Participantes } & \multicolumn{6}{|c|}{ Nível de severidade dos sintomas } \\
\hline & \multicolumn{2}{|c|}{ Ansiedade } & \multicolumn{2}{|c|}{ Depressão } & \multicolumn{2}{|c|}{ Desesperança } \\
\hline & Pré-teste & Pós-teste & Pré-teste & Pós-teste & Pré-teste & Pós-teste \\
\hline 1 & Moderada & Leve & Leve & Leve & Mínima & Mínima \\
\hline 2 & Moderada & Leve & Leve & Leve & Mínima & Mínima \\
\hline 3 & Mínima & Mínima & Mínima & Mínima & Mínima & Mínima \\
\hline 4 & Severa & Moderada & Severa & Moderada & Leve & Leve \\
\hline 5 & Moderada & Mínima & Moderada & Leve & Leve & Mínima \\
\hline 6 & Leve & Leve & Moderada & Leve & Mínima & Mínima \\
\hline 7 & Moderada & Leve & Mínima & Mínima & Mínima & Mínima \\
\hline 8 & Leve & Leve & Leve & Leve & Leve & Mínima \\
\hline 9 & Mínima & Mínima & Mínima & Mínima & Mínima & Mínima \\
\hline 10 & Mínima & Mínima & Mínima & Mínima & Mínima & Mínima \\
\hline 11 & Mínima & Leve & Leve & Leve & Mínima & Mínima \\
\hline
\end{tabular}

Não houve alterações no pós-teste para habilidades sociais, todos os jovens mantiveram o mesmo repertório que possuíam antes da intervenção. Pode-se considerar que tal resultado já era esperado, pelo fato de não ter sido considerado uma demanda crítica já no início e não ter sido um aspecto priorizado na intervenção. Cabe mencionar, ainda, que todos os participantes receberam devolutivas de seus resultados individualmente, momento no qual foram também feitos encaminhamentos nos casos que os demandavam.

Como se pode perceber, algumas importantes limitações devem ser levadas em conta ao analisar estes dados. Algumas dificuldades operacionais (troca de sala, de equipamento disponível, horários e datas), a assiduidade irregular, o curto tempo para algumas sessões e o fato de o pós-teste ter sido realizado logo após uma das provas, certamente comprometeram ou interferiram nos resultados apurados pelos instrumentos em pós-teste. Apesar de serem variáveis que muitas vezes fogem ao controle dos pesquisadores, novos esforços devem ser tomados para que diminuam em próximas experiências.

Feitas as devidas ressalvas, pode-se dizer que a intervenção desenvolvida parece ter potencial para auxiliar jovens que estejam prestando o vestibular a manejar seu stress e sua ansiedade. Mesmo as condições não tendo sido as ideais, os resultados apontaram a redução de sintomas de stress, ansiedade e depressivos, sugerindo efeitos positivos do programa, o que corrobora com achados da literatura (Knapp \& Beck, 2008; Moreno e Carvalho, 2014). Considerando que se tratou de uma intervenção grupal construída com base na TCC e no TCS, os resultados no pós-teste suportam os achados mencionados por Lipp (2017) para diminuição do stress. Do mesmo modo, quanto à ansiedade, estudo de Lantyer, Varanda, Souza, Padovani e Viana, (2016), que consistiu na intervenção grupal para controle da ansiedade baseada na Abordagem cognitivo-comportamental teve bons resultados na redução de sintomas. 
Mais contundentemente, o relato dos próprios participantes também parece indicar benefícios do programa, principalmente relacionados aos exercícios de relaxamento. Além desses, os relatos indicam que o simples fato de reconhecerem que estavam em uma situação geradora de desgaste emocional e, principalmente, de possuírem um espaço para falarem sobre isso, pôde ajudar a aliviar as pressões que estavam sofrendo.

\section{Considerações finais}

O presente artigo objetivou relatar experiência de aplicação de programa breve de intervenção para o manejo de stress e ansiedade em vestibulandos, bem como apresentar seus resultados preliminares. Frente aos resultados, pôde-se verificar o quanto a população de jovens que está prestando o vestibular precisa de atenção por parte de profissionais de saúde, educadores e pais. O programa apresentado, apesar das variáveis intervenientes, pareceu contribuir para a melhora dos sintomas dos participantes, o que indica seu potencial para novas experiências.

Questões como a quantidade pequena de participantes, reduzido número de sessões, evasão, horário e proximidade com as provas classificatórias são limitações que estiveram presentes neste estudo e precisam ser superadas em aplicações futuras, através da formalização dos acordos entre terapeuta, participantes e instituição. Além disso, como a descrição aqui realizada foi de uma versão piloto do programa, a próxima intervenção contará com mais sessões, visando conferir maior tempo para que assuntos complexos ou delicados possam ser melhor assimilados e elaborados. Futuras pesquisas podem incluir então maior número de sessões, amostra mais ampla, adoção de estudos estatísticos para verificação de diferenças significativas entre o pré e pós-teste e relações entre outras variáveis. Espera-se que os resultados encontrados nesta experiência possam se repetir e continuar a contribuir para a melhora no estado psicológico de jovens vestibulandos.

\section{Referências}

Araújo, M. C., França, N. M. d., Madeira, F. B., Sousa Júnior, I. d., Silva, G. C. B., Silva, E. d. F. R., \& Prestes, J. (2012). Efeitos do exercício físico sobre os níveis de stress em vestibulandos de Teresina-PI. Revista Brasileira de Ciência e Movimento, 20(3), 14-26. Recuperado em 15 fevereiro 2017, de https://portalrevistas.ucb.br/index.php/RBCM/ article/view/3094

Archer, A. B., Heumann, S., \& Luz Filho, S. S. D. (2011). Reflexões: Ansiedade frente à escolha profissional e a prova do vestibular. Extensio: Revista Eletrônica de Extensão, 8(11), 70-80. DOI: http://dx.doi. org/10.5007/1807-0221.2011v8n11p70

Caciari, B., \& Damazo, R. (2017). Adolescência e estresse: As estratégias de enfrentamento de um Grupo em Vulnerabilidade Social. MOMENTUM, 1(12), 53-82. Recuperado em 15 fevereiro 2017, de http://momentum.emnuvens.com.br/ momentum/article/view/26

Carrara, K., \& Betetto, M. d. F. (2009). Formação ética para a cidadania: uma investigação de habilidades sociais medidas pelo Inventário de Habilidades Sociais. Estudos de Psicologia, 26(3), 337-347. DOI: http://dx.doi.org/10.1590/ S0103-166X2009000300007

Cunha, J. A. (2001). Manual da versão em português das Escalas Beck. São Paulo: Casa do Psicólogo.

D’Avila, G. t., Krawulski, E., Veriguine, N. R., \& Soares, D. H. P. (2011). Acesso ao ensino superior eo projeto de "ser alguém" para vestibulandos de um cursinho popular. Psicologia \& Sociedade, 23(2), 350-358. DOI: http://dx.doi. org/10.1590/S0102-71822011000200016

D’Avila, G. T., \& Soares, D. H. P. (2003). Vestibular: fatores geradores de ansiedade na cena da prova. Revista Brasileira de Orientação Profissional, 4(1-2), 105-116. Recuperado em 15 fevereiro 2017, de http://pepsic.bvsalud.org/pdf/ rbop/v4n1-2/v4n1-2a10.pdf

Del Prette, Z. A. P., \& Del Prette, A. (2001). Inventário de Habilidades Sociais: Manual de aplicação, apuração e interpretação. São Paulo: Casa do Psicólogo.

Dias, L. S., Nazareno, E., Zanini, D. S., \& Mendonça, H. (2008). Vestibular e Adolescência: perspectivas teóricas e implicações sociopsicológicas. Revista Fragmentos de Cultura, 18(7/8), 625-636. DOI: http://dx.doi.org/10.18224/ frag.v18i4.712

Fagundes, P. R., Aquino, M. G. d., \& Paula, A. V. d. (2010). Pré-vestibulandos: Percepção do stress em jovens formandos do ensino médio. Akrópolis-Revista de Ciências Humanas da UNIPAR, 18(1), 57-69. Recuperado em 15 fevereiro 2017, de http://revistas.unipar.br/index.php/akropolis/article/view/3117

Guhur, M. d. L. P., Alberto, R. N., \& Carniatto, N. (2010). Influências biológicas, psicológicas e sociais do vestibular na 
adolescência. Roteiro, 35(1), 115-138. Recuperado em 15 fevereiro 2017, de http://editora.unoesc.edu.br/index.php/ roteiro/article/view/230

Knapp, P., \& Beck, A. T. (2008). Fundamentos, modelos conceituais, aplicações e pesquisa da terapia cognitiva. Revista Brasileira de Psiquiatria, 30(Supl II), S54-64. DOI: http://dx.doi.org/10.1590/S1516-44462008000600002

Kristensen, C. H., Schaefer, L. S., \& Busnello, F. d. B. (2010). Estratégias de coping e sintomas de stress na adolescência. Estudos de Psicologia, 27(1), 21-30. DOI: http://dx.doi.org/10.1590/S0103-166X2010000100003

Kuntze, M. M. (2013). Correlação entre o nivvel de ansiedade ea presença de dor de cabeça e cansaço muscular facial em estudantes de cursos pré-vestibular localizados na grande Florianópolis. (Trabalho de conclusão de curso), Universidade Federal de Santa Catarina, Florianópolis. Recuperado em 15 fevereiro 2017, de https://repositorio.ufsc. $\mathrm{br} / \mathrm{xmlui} /$ handle/123456789/100306?show=full

Lantyer, A. d. S., Varanda, C. C., Souza, F. G. d., Padovani, R. D. C., \& Viana, M. D. B. (2016). Ansiedade e qualidade de vida entre estudantes universitários ingressantes: avaliação e intervenção. Revista Brasileira de Terapia Comportamental e Cognitiva, 18(2), 4-19. Recuperado em 15 fevereiro 2017, de http://www.usp.br/rbtcc/index.php/ RBTCC/article/view/880

Lins, M. F. N., \& Viana, M. T. (2016). Vestibular e as repercussões associadas à ansiedade dos candidatos. Repositório digital ASCES. Recuperado em 15 fevereiro 2017, de http://repositorio.asces.edu.br/handle/123456789/638

Lipp, M. E. N. (2000). Manual do inventário de sintomas de stress para adultos de Lipp (ISSL). São Paulo: Casa do Psicólogo.

Lipp, M. E. N. (2012). Relaxamento para todos. Campinas - SP: Papirus Editora.

Lipp, M. E. N. (2017). O Treino de Controle do Estresse em grupo: Um modelo da TCC. In: C. B. Neufeld, \& B. P. Rangé (Orgs). Terapia cognitivo-comportamental em grupo: das evidências à prática. Porto Alegre, RS: Artmed.

Maria, W. B., Guimarães, A. C. d. A., \& Matias, T. S. (2009). Estilo de vida de adolescentes de escolas públicas e privadas de Florianópolis-SC. Revista da Educação Física/UEM, 20(4), 615-623. Recuperado em 15 fevereiro 2017, de http://www.periodicos.uem.br/ojs/index.php/RevEducFis/article/view/6943

Marques, C. P. (2014). Estresse, comportamento de risco e atividade física de estudantes pré vestibulandos. Tese de Doutorado, Universidade Federal do Paraná, Curitiba. Recuperado em 15 fevereiro 2017, de http://www.acervodigital. ufpr.br/handle/1884/35733?show=full

Moreira, L. C. d. O., \& Bastos, P. R. H. d. O. (2015). Prevalência e fatores associados à ideação suicida na adolescência: revisão de literatura. Psicologia Escolar e Educacional, 19(3), 445-453. DOI: http://dx.doi. org/10.1590/2175-3539/2015/0193857

Moreno, A. L., \& Carvalho, R. G. N. D. (2014). Terapia cognitivo-comportamental breve para sintomas de ansiedade e depressão. Revista Brasileira de Terapias Cognitivas, 10(2), 70-75. DOI: http://dx.doi. org/10.5935/1808-5687.20140012

Oliveira, C. T. D., Dias, A. C. G., \& Piccoloto, N. M. (2013). Contribuições da terapia cognitivo-comportamental para as dificuldades de adaptação acadêmica. Revista Brasileira de Terapias Cognitivas, 9(1), 10-18. DOI: http://dx.doi. org/10.5935/1808-5687.20130003

Paggiaro, P. B. S., \& Calais, S. L. (2009). Stress e escolha profissional: um difícil problema para alunos de curso prévestibular. Contextos Clínicos, 2(2), 97-105. DOI: 10.4013/ctc.2009.22.04

Pellegrini, C. F. d. S., Calais, S. L., \& Salgado, M. H. (2012). Habilidades sociais e administração de tempo no manejo do stress. Arquivos Brasileiros de Psicologia, 64(3), 110-129. Recuperado em 15 fevereiro 2017, de http://pepsic. bvsalud.org/pdf/arbp/v64n3/v64n3a08.pdf

Resende, C., Santos, E., Santos, P., \& Ferrão, A. (2013). Depressão nos adolescentes: mito ou realidade? Nascer e Crescer, 22(3), 145-150. Recuperado em 15 fevereiro 2017, de http://www.scielo.mec.pt/pdf/nas/v22n3/v22n3a03.pdf

Rodrigues, D. G., \& Pelisoli, C. (2008). Ansiedade em vestibulandos: um estudo exploratório. Revista de Psiquiatria Clínica, 35(5), 171-177. DOI: http://dx.doi.org/10.1590/S0101-60832008000500001

Santos, F. O. d. (2011). Comportamentos em saúde: hábitos alimentares, composição corporal, atividade fisica, ansiedade, em alunos no periodo pré-vestibular em Campina Grande-PB. (Trabalho de conclusão de curso), Universidade Estadual da Paraíba, Campina Grande. Recuperado de: http://dspace.bc.uepb.edu.br:8080/xmlui/ handle/123456789/947

Silva, L. S. D. e., \& Zanini, D. S. (2011). Coping e saúde mental de adolescentes vestibulandos. Estudos de Psicologia, 16(2), 147-154. DOI: http://dx.doi.org/10.1590/S1413-294X2011000200005 
Souza, L. C. d., Bonganha, V., Chacon-Mikahil, M. P. T., \& Madruga, V. A. (2013). Efeitos de um programa de condicionamento físico sobre o nível de stress em integrantes universitárias. Conexões: Revista da Faculdade de Educação Física da UNICAMP, 11(1), 42-56. Disponível em: https://periodicos.sbu.unicamp.br/ojs/index.php/ conexoes/article/view/8637630

Tomasik, M. J., \& Salmela-Aro, K. (2012). Knowing when to let go at the entrance to university: Beneficial effects of compensatory secondary control after failure. Motivation and Emotion, 36(2), 170-179. DOI: http://dx.doi. org/10.1007/s11031-011-9246-5

Villamarín, A. J. G. (2000). Como Superar a Barreira do Vestibular. Porto Alegre: Editora AGE Ltda.

Recebido: $15 / 02 / 2017$

$1^{a}$ revisão: $18 / 10 / 2017$

Aceite final: 20/12/2017

Sobre as autoras

Carla Cristina Daolio é Psicóloga, formada pela Faculdade de Filosofia, Ciências e Letras de Ribeirão Preto (FFCLRP) da Universidade de São Paulo, campus de Ribeirão Preto. Atualmente realiza prática privada.

Carmem Beatriz Neufeld é Professora associada do Departamento de Psicologia da Faculdade de Filosofia, Ciências e Letras de Ribeirão Preto da Universidade de São Paulo. Bolsista produtividade CNPq. Coordenadora do Laboratório de Pesquisa e Intervenção Cognitivo-Comportamental - LaPICC. 